\title{
Development of Mobile Website-based Alumni Survey (moWAS) for Assessment of Programme Educational Objectives (PEOs)
}

\author{
${ }^{1}$ Shamsul Mohamad, ${ }^{2}$ Rafizah Mohd Hanifa, ${ }^{3}$ Zarina Tukiran, ${ }^{4}$ Afandi Ahmad \\ ${ }^{1,3,4}$ Faculty of Electrical and Electronic Engineering, UTHM \\ ${ }^{2}$ Center for Diploma Studies, UTHM
}

\begin{abstract}
Assessment and review of Programme Educational Objectives (PEOs) are important parts in Outcome Based Education (OBE). Currently, the assessment is conducted on a paper-based survey to the alumnis. Since the survey data is entered manually into excel-based OBE system, it creates additional work for the committee incharge. Mobile website-based alumni survey (moWAS) has been designed and developed to broaden the accessibility to Alumni of the Faculty of Electrical and Electronic Engineering. Data gathered through this survey will be analysed to ensure the PEOs fulfill the requirements of the Engineering Accreditation Council Malaysia (EAC). This paper addresses the development and the functionality of moWAS.
\end{abstract}

Keywords: mobile web application, online survey, program educational outcomes

\section{Introduction}

For the past few years, the internet has been used by many companies all over the world in conducting all sorts of studies. Regardless of whether it is a market or scientific research, the online survey has been a faster way of collecting data directly from respondents, as compared to other survey methods such as paper-and-pencil method and personal interviews [1].

A survey is also used by the Outcome Based Education (OBE) committee of the Bachelor Degree in Electrical Engineering (BEE) programme as one of the tool to measure the attainment of PEOs from the alumni themselves. The assessment is currently conducted using paper-based survey and the survey data is entered manually into the excel-based OBE system. This practice creates additional workload to the committee. Therefore, as an effort to ease the burden and increase the effiency in producing the results for analysis, the mobile websitebased OBE system or shortly known as mOBEs was proposed. The mOBEs comprises of three subsystems: PEOs, Program Outcomes (POs) and Course Learning Outcomes (CLOs). The CLOs and POs subsystems in assisting the committee to assess and review the attainments of the CLOs and POs have been discussed in [2]. Therefore, this paper focuses on the development of mobile website-based alumni survey (moWAS) which will cover the PEOs for alumnis.

The paper is organised as follows. The design of moWAS to be used in mobile devices to deliver survey to 
alumni is discuss in the next section. The implementation of moWAS and its functionality are described in section three. The conclusion and future works are briefly described in the last section.

\section{Proposed Design}

The Faculty's PEOs are currently being assessed using the following assessment tools [3]: (1) Reports and minutes of meeting with Industrial Advisors Panel (IAP), (2) Reports and minutes of meeting with External Examiner, (3) Alumni survey and (4) Employer survey. The current framework of PEOs as shown in Fig. 1 is adopted to design the moWAS. The discussion in this paper is primarily on the gray boxes.

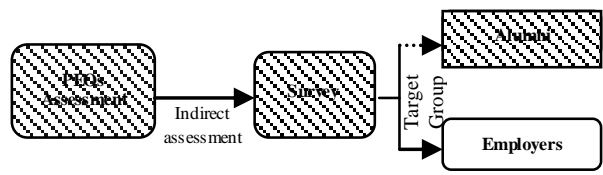

Fig. 1: PEOs Assessment Framework

The ultimate goal of moWAS is to resolve some of the issues that arise in paper-based system related to data gathering and analyzing. To ensure the goal is achieved, moWAS is designed for viewing contents on mobile phones to make it accessible to wider targeted users as well as being user friendly. moWAS combines the versatility of the web with the functionality of touch-enabled devices [4]. moWAS will not be installed on the device; rather, they are accessed via a Web browser. The moWAS model is represented using Unified Modeling Language (UML). In Unified Modeling Language (UML), a use case diagram describes the interactions of users and components in a software system [5].

The functionality of moWAS is shown in Fig. 2 using the use case diagram. In order to make moWAS easy to use, the users are treated as role players. A single user, called the administrator, has the right to see the details of the survey including the graphs showing the responses from the respondents. As for the alumni, they need to register their details to be eligible to do the survey. The system brings up the proper interface for an individual user based on their role(s). Fig. 3 shows the flowchart for moWAS.

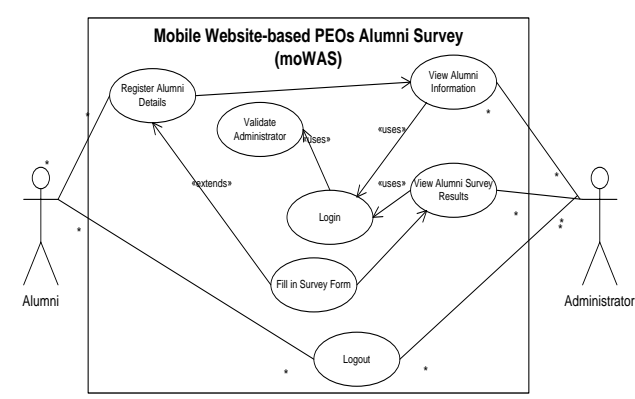

Fig. 2: Use Case Diagram for moWAS

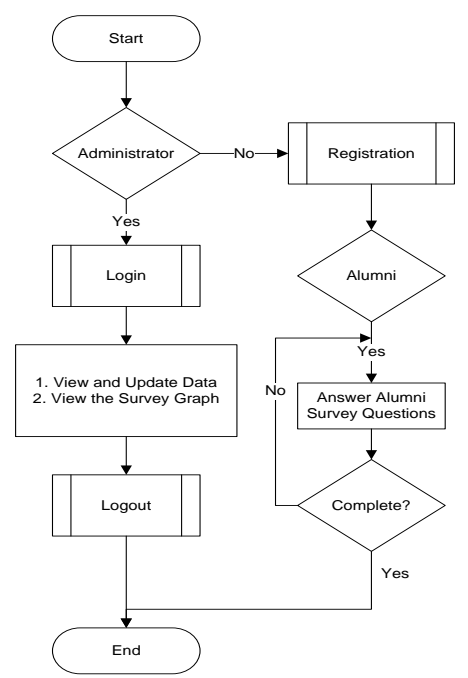

Fig. 3: Flow Chart for moWAS

\section{System implementation}

moWAS takes advantage of the free and open source technologies in its implementation. The free and open source tool called jQuery, written in JavaScript is intended to handle the 
administration of MySQL, with the use of AJAX. jQuery can run on Internet Explorer 9+, Mozilla Firefox, Google Chrome, Safari and Opera [6]. Fig. 4 shows the main interface of moWAS. There are two options: About PEOs and PEOs Survey. The About PEOs option explains the PEOs in detail and how it will be assessed. This information is important for respondents to understand better what PEOs is all about before starting to answer the survey. As for the PEOs Survey, the respondents will be led to the survey questions. However, before doing so, they have to register their details as shown in Fig. 5.

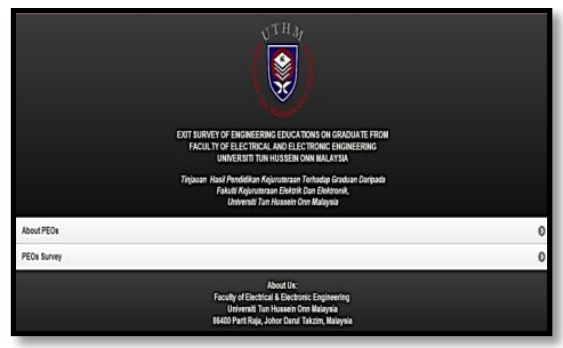

Fig. 4: moWAS Main Interface

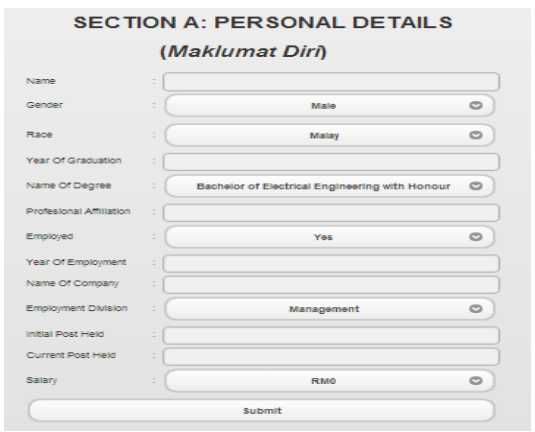

Fig. 5: Details of Alumni

Once they have registered, they can start answering the survey. The example of survey questions for alumni is shown in Fig. 6. The data entered are added into the database. As an administrator, he/she can view the graphical analysis based on the questions answered by respondents as shown in Fig. 7. This is much easier for decision making compared to if this was done manually.

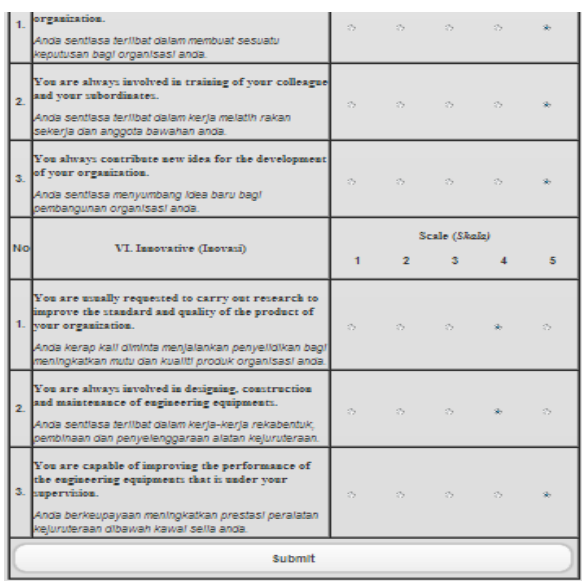

Fig. 6: Sample of questions

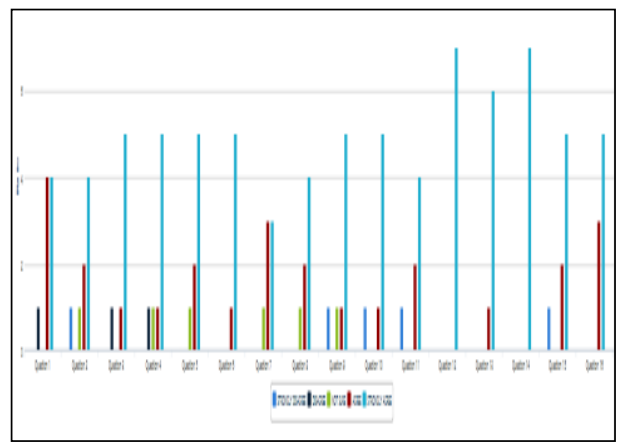

Fig. 7: Analysis graphs answered by respondents

\section{Conclusion and Future work}

PEOs are statements that indicate the expected achievements of graduates on the program in terms of their career and professional life after graduation. These relate to the skills, knowledge and behavior that students acquire in their learning sessions throughout the program. The feedback from the alumni will enable the OBE Committee of the faculty to reflect, discuss and suggest changes and/or modifications to the objectives, if 
necessary. Thus, the proliferation of the use of Internet, low cost of hardware and the enhancements in wireless communication enabled us to develop moWAS in a more organized and efficient way. Since the developed moWAS focuses on gathering and collecting data from alumni, a further step will be to design for the employers' survey and generate the final PEOs report.

\section{Acknowledgement}

The authors would like to thank Universiti Tun Hussein Onn Malaysia for funding this research under Short Term Grant Phase 1/2012 (Vote 0991).

\section{References}

[1] Sarah Mae Sincero (Oct 16, 2012). Online Surveys. Retrieved Jun 08, 2013 from Explorable.com: http://explorable.com/online-surveys

[2] Shamsul et. al. An Evaluation of Assessment Tools in Outcome-based Education: A Way Forward. Journal of Education and Vocational
Research. Vol. 3, No. 11, pp. 336343, Nov 2012 (ISSN 2221-2590)

[3] OBE Implementation Guidebook, Faculty of Electronic \& Electronic Engineering 2010, prepared by OBE Committee, FKEE.

[4] Sybrant Technologies (P) Ltd (n.d.), Mobile Application Development Framework. Retrieved June 13, 2013, from http://www.sybrant.com/mobileapplication-development-framework

[5] Broy, M., \& Cengarle, M. (2011). UML formal semantics: lesson learned. Software \& System Modeling, 10(4), 441-446. doi:10.1007/s10270-011-0207-y

[6] jQuery Foundation (October 2, 2012), jQuery Mobile. Retrieved June 10, 2013, from http://en.wikipedia.org/wiki/JQuery_ Mobile 